\title{
Close and distant reprocessing media in Mkn 509 studied with BeppoSAX
}

\author{
A. De Rosa ${ }^{1}$, L. Piro ${ }^{1}$, G. Matt ${ }^{2}$, and G. C. Perola ${ }^{2}$ \\ ${ }^{1}$ Istituto di Astrofisica Spaziale e Fisica Cosmica, C.N.R., Via Fosso del Cavaliere, Roma, Italy \\ 2 Dipartimento di Fisica, Università degli Studi "Roma Tre", Via della Vasca Navale 84, 00146 Roma, Italy \\ Received 4 July 2003 / Accepted 23 September 2003
}

\begin{abstract}
We present the broad band analysis of two BeppoSAX observations of the Seyfert 1 Mkn 509. In 2000 the source was in a typical flux state, $F_{2-10 \mathrm{kev}}=2.7 \times 10^{-11} \mathrm{erg} \mathrm{cm}^{-2} \mathrm{~s}^{-1}$, while in 1998 it was found in a high flux state, $F_{2-10 \mathrm{keV}}=$ $5.7 \times 10^{-11} \mathrm{erg} \mathrm{cm}^{-2} \mathrm{~s}^{-1}$. A comparison between the two states shows a energy-dependent flux variation of about a factor of three and a factor of two in the LECS (0.15-3 keV) and MECS (1.5-10 keV), respectively, while in the PDS (13-200 keV) the difference is marginal. A soft excess, a narrow iron line and a Compton reflection hump above $10 \mathrm{keV}$ are clearly apparent in the residuals after fitting the spectra with a simple power law. We tested two alternative models. In the first the iron line and the high energy bump are well reproduced by reprocessing in a cold and Compton thick material. The intensity of the iron line (also consistent with a Chandra measurement) as well as the normalization of the reflection hump are consistent with a constant in the two epochs: this, combined with the fact that the line is narrow as observed by Chandra, suggests a common origin from distant and optically thick matter. This model further requires a component to model the soft excess: the empirical choice of two black bodies accounts well for the excess in both observations; their combined strength was a factor of about three higher in the high than in the low flux state defined above. However, the relative contribution of the soft excess is higher in the low flux state. In the second model we attempted to reproduce all spectral features, except for the narrow cold line, with reflection from an ionized disc. This model is successful only in the high flux state, but it fails in the low flux state, when the soft excess is only partially accounted for. In either model, the slope of the power law is greater in the high than in the low flux state, $(\Delta \Gamma \sim 0.2)$, in agreement with a behaviour known to be shared by several objects of the same type.
\end{abstract}

Key words. galaxies: individual: Mkn 509 - galaxies: Seyfert - X-rays: galaxies

\section{Introduction}

The most popular model for X-ray emission in AGN envisages a hot, optically-thin plasma (the corona) which up-scatters to X-ray energies the soft photons produced in an optically thick accretion disc (e.g. Haardt \& Maraschi 1993). The disc, in its turn, reprocesses and re-emits part of the Comptonized flux, so producing the characteristic reprocessing features, the Compton reflection hump and the fluorescent iron $\mathrm{K} \alpha$ line. The presence of a broad component of the iron line (Fabian et al. 2000) in the X-ray spectra of Seyfert galaxies was first observed by ASCA (Tanaka et al. 1995; Nandra et al. 1997; Yaqoob et al. 2002) and confirmed by BeppoSAX (Guainazzi et al. 1999) and by XMM-Newton (Fabian et al. 2002; Wilms et al. 2001) observations. However, Chandra and $X M M-N e w t o n$ observations clearly indicate that a narrow component, likely originating in distant matter, is also almost always present (Reeves et al. 2001; Pounds et al. 2001; Matt et al. 2001; Yaqoob et al. 2001; Kaspi et al. 2001; Pounds et al. 2003). The BLR and the optically thick torus are the best candidate regions to produce this narrow component. In the latter, the matter is likely to be Compton-thick

Send offprint requests to: A. De Rosa,

e-mail: derosa@rm.iasf.cnr.it
$\left(N_{\mathrm{H}}>10^{24} \mathrm{~cm}^{-2}\right)$ and a Compton reflection component is also expected (Ghisellini et al. 1994; Matt et al. 2003), as already observed in NGC 4051 (Guanazzi et al. 1998) and NGC 5506 (Matt et al. 2001).

In this paper we present the 2000 BeppoSAX observations of the Seyfert 1 galaxy Mkn 509, and compare it with the 1998 observation, already discussed by Perola et al. (2000). This source has a typical X-ray luminosity (in the $2-10 \mathrm{keV}$ energy range) of $3 \times 10^{44} \mathrm{erg} \mathrm{s}^{-1}\left(H_{0}=50 \mathrm{~km} \mathrm{~s}^{-1}\right.$ $\mathrm{Mpc}^{-1}$ ) at $z=0.034$, with a Galactic absorption column of $N_{\mathrm{H}}^{\mathrm{Gal}}=4.4 \times 10^{20} \mathrm{~cm}^{-2}$ (Murphy et al. 1996). A soft excess in the X-ray spectra was observed by a ROSAT and Ginga simultaneous observation (Pounds et al. 1994), confirming the previous HEAO-1 (Singh et al. 1985) and EXOSAT finding (Morini et al. 1987). The ASCA observation, however, suggested a warm absorber component in Mkn 509 rather than a true soft excess (Reynolds 1997; George et al. 1998), but BeppoSAX (Perola et al. 2000) and XMM-Newton (Pounds et al. 2001) confirmed the soft X-ray excess below $1 \mathrm{keV}$, and showed also the presence of a narrow iron line (already detected by Ginga and $A S C A$ ) and a Compton reflection bump above $10 \mathrm{keV}$ (Singh et al. 1990). 

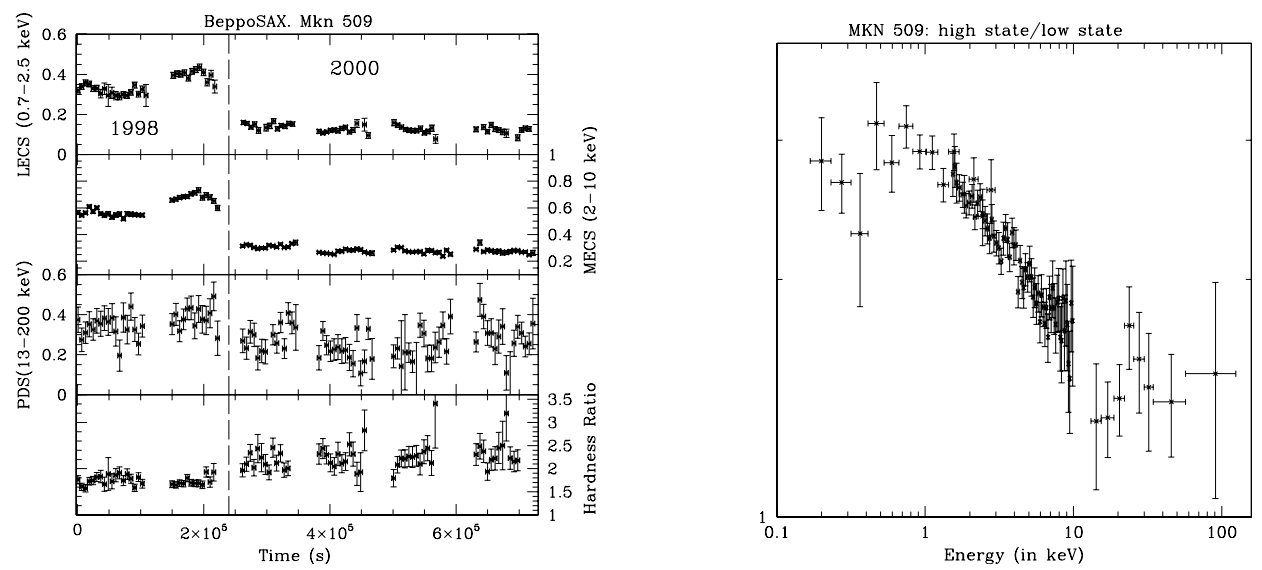

Fig. 1. Left panel. Lightcurves and hardness ratio MECS(2-10 keV)/LECS(0.7-2.5 keV) of the BeppoSAX observations (the binsize is $6000 \mathrm{~s})$. The time intervals between the different looks in 1998 and 2000 are fake. Right panel. Spectral ratio between the high (1998) and low (2000) flux state.

The observations and data reduction are described in Sect. 2. A model-independent variability study is presented in Sect. 3. The spectral analysis is reported in Sect. 4 and discussed in Sect. 5. Our conclusions are drawn in Sect. 6.

\section{Observations and data reduction}

Mkn 509 was observed by BeppoSAX (Boella et al. 1997) two times in 1998 (May 18, October 11) and four times in 2000 (November 3-8-18-24). The LECS, MECS and PDS data reduction followed the standard procedure (Fiore et al. 1999). The PDS spectra were filtered with variable rise time. We extracted the spectrum within circular regions around the source centroid with radii of $4^{\prime}$ and $8^{\prime}$ for the MECS and LECS, respectively. The background was extracted from event files of source-free regions ("blank fields"). Spectral models were fitted to the data using the XSPEC package V11.2. All quoted uncertainties correspond to the $90 \%$ confidence level for one interesting parameter $\left(\Delta \chi^{2}=2.71\right)$. The models included a normalization factor for each instrument to take into account intercalibrations. We fixed the PDS/MECS normalization to 0.8 while the LECS/MECS normalization ratio was between 0.7 and 1 (Fiore et al. 1999).

In Fig. 1 we show the LECS (0.7-2.5 keV), MECS (2$10 \mathrm{keV}$ ) and PDS (13-200 keV) light curves (the binsize is $6000 \mathrm{~s}$ ), and in the last panel the hardness ratio MECS (2$10 \mathrm{keV}) / \mathrm{LECS}(0.7-2.5 \mathrm{keV})$. The source does not show significant spectral variations within the two sets of observations, thus we combined the two in 1998 as well as the four in 2000. The journal of the combined observations is shown in Table 1. In 1998 Mkn 509 was in a relatively bright flux state, $F_{2-10 \mathrm{keV}}=5.66 \times 10^{-11} \mathrm{erg} \mathrm{cm}^{-2} \mathrm{~s}^{-1}$, while in 2000 it was at a more typical flux level, $F_{2-10 \mathrm{keV}}=2.7 \times 10^{-11} \mathrm{erg} \mathrm{cm}^{-2} \mathrm{~s}^{-1}$.

\section{Spectral ratio}

The two states are characterized by different spectral shapes, as immediately revealed, in a model-independent way, by the ratio of the spectra (right panel of Fig. 1):

1) In the $1-10 \mathrm{keV}$ energy range a change in the slope is apparent (with a pivot point around 70-80 keV), the steeper spectrum corresponding to the larger flux state. Since in this band the incidence of absorption, reflection component and soft excess is negligible, the change must be associated with the primary, power law component.

2) Below $1 \mathrm{keV}$ the ratio suggests the presence of another component, which varied in a different way with respect to the power law.

3) In the $10-100 \mathrm{keV}$ range the ratio is consistent with a constant value. This one being the region where Compton reflection is important, this result suggests an approximately constant intensity in this additional component, despite the change of the primary flux.

In the next section we will test these results with a detailed spectral analysis.

\section{Broad band spectral analysis}

\subsection{Cold disc reflection model}

Mkn 509 is known to possess a soft excess, as observed by EXOSAT (Turner et al. 1991), ROSAT (Pounds et al. 1994), BeppoSAX (Perola et al. 2000) and XMM-Newton (Pounds et al. 2001). We first excluded the energy range where the excess is present by fitting the 1998 and 2000 spectra from 3 to $100 \mathrm{keV}$ with a power law multiplied by $\exp \left(-E / E_{\mathrm{c}}\right)$, a cold reflection component (PEXRAV, Magdziarz \& Zdziarski 1995, with inclination angle fixed to $i=30^{\circ}$ ) and a Gaussian component to model the iron line. With the intrinsic width of the line as a free parameter, the fit yields an unacceptably large value of $\sigma \sim 2 \mathrm{keV}$ in both spectra, that was likely due to a bad description of the continuum at the line energy. This parameter is however basically unconstrained and in fact, with a width fixed to $0.1 \mathrm{keV}$, the fit is equally good. We therefore adopted this value; the results are reported for both epochs in Table 2.

The $\chi^{2}$ is fully acceptable in both observations $\left(\left(\chi^{2} / \text { d.o.f. }\right)_{2000}=60 / 66,\left(\chi^{2} / \text { d.o.f. }\right)_{1998}=54 / 66\right)$. The narrow line energy is consistent in both spectra with neutral iron, with an intensity that does not appear to vary significantly between the low and the high flux states.

A strong soft excess at $E<3 \mathrm{keV}$ appears when the 3-100 keV best fit spectra are extrapolated to the LECS energy 
Table 1. Mkn 509 by BeppoSAX. Journal of the observations in 1998 (sum of two) and 2000 (sum of four).

\begin{tabular}{ccccc}
\hline \hline OBS & $\begin{array}{c}\text { LECS }(0.15-3 \mathrm{keV}) \\
\left(\mathrm{s}^{-1}\right)\end{array}$ & $\begin{array}{c}\text { MECS }(1.5-10 \mathrm{keV}) \\
\left(\mathrm{s}^{-1}\right)\end{array}$ & $\begin{array}{c}\text { PDS (13-200 keV) } \\
\left(\mathrm{s}^{-1}\right)\end{array}$ & $\begin{array}{c}t_{\exp } \text { (MECS) } \\
(\mathrm{s})\end{array}$ \\
\hline 1998 & $0.464 \pm 0.003$ & $0.724 \pm 0.003$ & $0.77 \pm 0.03$ & 87834 \\
2000 & $0.167 \pm 0.002$ & $0.325 \pm 0.001$ & $0.58 \pm 0.03$ & 152777 \\
\hline
\end{tabular}

Table 2. Best fit spectra between 3-100 keV in the low (2000) and high (1998) flux state of Mkn 509.

\begin{tabular}{cccccccc}
\hline \hline & $\Gamma$ & $E_{\mathrm{c}}(\mathrm{keV})$ & $R$ & ${ }^{\circ} I_{\mathrm{Fe}}$ & $E W_{\mathrm{Fe}}(\mathrm{eV})$ & $E_{\mathrm{Fe}}^{\mathrm{obs}}(\mathrm{keV})$ & $\chi^{2} /$ d.o.f. \\
\hline 2000 & $1.59_{-0.06}^{+0.07}$ & $83_{-20}^{+47}$ & $0.86_{-0.31}^{+0.40}$ & $2.8_{-1.0}^{+1.8}$ & $96 \pm 60$ & $6.33_{-0.15}^{+0.19}$ & $60 / 66$ \\
\hline 1998 & $1.80_{-0.06}^{+0.05}$ & $110_{-70}^{+40}$ & $0.64_{-0.26}^{+0.33}$ & $3.3_{-1.7}^{+1.6}$ & $57 \pm 27$ & $6.37_{-0.17}^{+0.19}$ & $54 / 66$ \\
\hline
\end{tabular}

* In $10^{-5} \mathrm{ph} \mathrm{cm}^{-2} \mathrm{~s}^{-1}$ at the line energy.
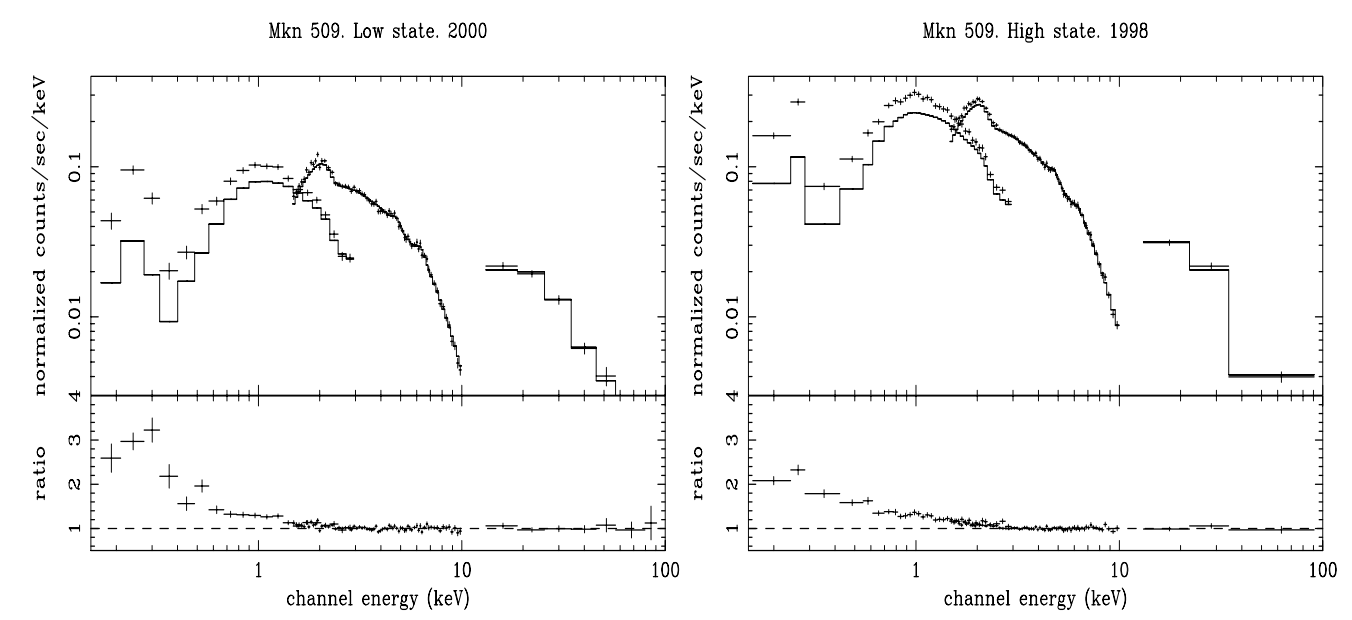

Fig. 2. Hard X-ray spectral fit for $E>3 \mathrm{keV}$ extrapolated to $0.15 \mathrm{keV}$ in the BeppoSAX-LECS energy band. A soft X-ray excess is present. The ratios data/model are shown as this spectral component seems have a larger contribution in the low flux state of Mkn 509 (left panel, see discussion in the text for details).

band (see Fig. 2). With the cold reflection factor $R$, the energy and width of the line fixed at the values from the previous fit, we first attempted to model the soft excess with one black body component: the result is not $\operatorname{good}\left(\left(\chi^{2} / \text { d.o.f. }\right)_{2000}=186.7 / 143\right.$, $\left.\left(\chi^{2} / \text { d.o.f. }\right)_{1998}=166.1 / 143\right)$; a much better result in both observations is obtained with the addition of a second black body (the probability of exceeding $F$ with the addition of two parameters is greater than 99.9 per cent in both spectra). It is worth recalling here that Pounds et al. (2001) resorted to three black bodies to model the soft excess observed with XMM-Newton.

When we try to substitute the two black body components with a disc black body model, the fit becomes worse in both spectra $\left(\chi^{2} /\right.$ d.o.f. $=177 / 140$ in 2000 and $\chi^{2} /$ d.o.f. $=150 / 140$ in 1998).

We also tried, without success, to add, instead of the second black body, two edges at the energies of OVII $(0.74 \mathrm{keV})$ and OVIII $(0.87 \mathrm{keV})$, whose optical depths are consistent with zero in both spectra.
The best fit spectra with two black bodies are summarized in Table 3, and the data/model ratio is shown in the left panel of Fig. 3. The intensities of the iron line in the two flux states are consistent with a constant value: $I_{\mathrm{Fe}}^{2000}=(2.8 \pm 1.1) \times$ $10^{-5} \mathrm{ph} \mathrm{cm}^{-2} \mathrm{~s}^{-1}$ and $I_{\mathrm{Fe}}^{1998}=(3.4 \pm 2.0) \times 10^{-5} \mathrm{ph} \mathrm{cm}^{-2} \mathrm{~s}^{-1}$.

If, following the claim by Pounds et al. (2001), a second iron line is added to the previous model to account for a possible contribution from an ionized component, the fit improves marginally (the probability of exceeding $F$ with the addition of two parameters, the energy and intensity of the line, is 95 and 93 per cent in the low and high flux state respectively). In both spectra the (poorly constrained) line energy is consistent with $\mathrm{H}$-like iron, and the equivalent widths are $\sim 60 \mathrm{eV}$ and $\sim 45 \mathrm{eV}$ in the low and high flux states, respectively.

The results shown in Table 3 quantitatively confirm our first comment in Fig. 1, with a change of the spectral index $\Delta \Gamma \sim 0.2$ (see Fig. 4). This behaviour is consistent with that observed in other Seyfert 1 galaxies (NGC 4151: Perola et al. 1986; 
Table 3. Cold disc reflection model. Broad band best fit spectra.

\begin{tabular}{cccccccccc}
\hline \hline & $\Gamma$ & $E_{\mathrm{c}}(\mathrm{keV})$ & $R$ & $k T_{\mathrm{BB}}^{1}(\mathrm{eV})$ & $k T_{\mathrm{BB}}^{2}(\mathrm{eV})$ & ${ }^{\star} I_{\mathrm{Fe}}$ & $E W_{\mathrm{Fe}}(\mathrm{eV})$ & $\chi^{2} /$ d.o.f. & Null hyp prob \\
\hline 2000 & $1.60 \pm_{0.06}^{0.07}$ & $84_{-19}^{+36}$ & $0.90 \pm_{0.35}^{0.50}$ & $71_{-7}^{+6}$ & $243_{-47}^{+27}$ & $2.8 \pm 1.1$ & $94 \pm 37$ & $157.4 / 138$ & 0.124 \\
\hline 1998 & $1.82 \pm 0.06$ & $115_{-43}^{+92}$ & $0.70_{-0.25}^{+0.45}$ & $71_{-8}^{+6}$ & $260 \pm 25$ & $3.4 \pm 2.0$ & $62 \pm 36$ & $127.8 / 138$ & 0.721 \\
\hline
\end{tabular}

* In $10^{-5} \mathrm{ph} \mathrm{cm}^{-2} \mathrm{~s}^{-1}$ at the line energy.

Table 4. The ionized disc reflection model. Broad band best fit spectra. The disc radial emissivity law is $\epsilon(r) \propto r^{\beta}$, with $\beta=-3$. The inner and outer radii were kept fixed respectively to $r_{\text {in }}=6 r_{\mathrm{g}}$ and $r_{\mathrm{out}}=10^{3} r_{\mathrm{g}}$. with $r_{g}=\mathrm{Gm} / \mathrm{c}^{2}$ the gravitational radius.

\begin{tabular}{cccccccc}
\hline \hline & $\Gamma$ & $\log (\xi)$ & $R$ & ${ }^{\star} I_{\mathrm{Fe}}^{\text {narrow }}$ & $E W_{\mathrm{Fe}}^{\text {narrow }}(\mathrm{eV})$ & $\chi^{2} /$ d.o.f. & Null hyp prob \\
\hline 2000 & $1.70 \pm 0.02$ & $1.55_{-0.04}^{+0.12}$ & $1.14 \pm 0.15$ & $1.6 \pm 0.9$ & $62 \pm 35$ & $183.2 / 143$ & 0.013 \\
\hline 1998 & $1.89 \pm 0.02$ & $1.55_{-0.22}^{+0.11}$ & $0.75_{-0.10}^{+0.14}$ & $2.3 \pm 1.6$ & $45 \pm 30$ & $155.3 / 143$ & 0.228 \\
\hline
\end{tabular}

* In $10^{-5} \mathrm{ph} \mathrm{cm}^{-2} \mathrm{~s}^{-1}$ at the line energy.

Piro et al. 1998; NGC 5548: Nicastro et al. 2000; IC 4329a: Done et al. 2000; NGC 7469: Nandra et al. 2000; MCG +630-15: Vaughan \& Edelson 2001; NGC 3783: De Rosa et al. 2002a), and with the $F_{\mathrm{x}}$ vs. $\alpha$ relation expected in a Comptonization model for the intrinsic continuum emission (Petrucci et al. 2000 and references therein).

With regard to our second comment in Fig. 1, we note that the strength of the two black body soft excess, in the $0.25-2 \mathrm{keV}$ range, is a factor of about three times larger in the 1998 epoch than in the 2000 epoch.

With regard to our third comment in Fig. 1, the normalization of the Compton reflection component (which we verified to depend very weakly on the value of the slope of the incident radiation) turns out to be $A=(2.3 \pm 0.6) \times 10^{-2}$ in 1998 and $A=\left(2.0_{-0.6}^{+0.4}\right) \times 10^{-2}$ in 2000 , consistent with a constant value, very much like the intensity of the cold iron line.

The fact that, as shown in Fig. 4, its relative normalization appears also constant (that, within the large errors, could suggest a reflection component which follows the intrinsic changes), is only the consequence of the $F_{\mathrm{x}}$ vs. $\alpha$ relation holding in the flux range that we happened to cover.

\subsection{The ionized disc reflection model}

The possible presence of a line from highly ionized iron (as claimed by Pounds et al. 2001) is suggestive of reflection from an ionized accretion disc. In this case the reflected component can be strong below $\sim 1 \mathrm{keV}$ and could significantly contribute to the observed soft excess in Mkn 509.
We therefore fitted the spectra with a reflection model that takes into account ionization of the accretion disc (Ross \& Fabian 1993). The most important quantity in determining the shape of the reflected continuum is the ionization parameter $\xi=4 \pi F_{\mathrm{x}} / n_{\mathrm{H}}$, where $F_{\mathrm{x}}$ is the $\mathrm{X}$-ray flux (between $0.01-100 \mathrm{keV}$ ) illuminating a slab of gas with solar abundance and constant hydrogen number density $n_{\mathrm{H}}=10^{15} \mathrm{~cm}^{-3}$. The incident flux is assumed to be a power law with spectral photon index $\Gamma$ and a sharp high energy cut-off at $100 \mathrm{keV}$. The reflected spectrum, which includes the $\mathrm{Fe} \mathrm{K} \alpha$, is multiplied by $R$ and added to the primary continuum. Different values of $\xi$ indicate different ionized states, affecting the strength and width of the Fe line (Matt et al. 1993; Matt et al. 1996), and of the absorption edges. We applied to the reflection spectrum the relativistic smearing for the Schwarzschild metric assuming an emissivity law $r^{\beta}$, with $\beta=-3$ and inner and outer radii fixed to $6 r_{\mathrm{g}}$ and $1000 r_{\mathrm{g}}$. Finally, a narrow and cold iron line was further added to the fitting model.

The best fit parameters are given in Table 4, and the data/model ratios are plotted in the right panel of Fig. 3. Notably, in the high flux level spectrum (1998) the ionized reflection accounts for the soft X-ray emission, and there is no need for an additional soft component. The $\chi^{2}$ is acceptable, albeit not as good as with the alternative model; the same is not true in the low flux level spectrum (2000), where in particular the residuals both below $1 \mathrm{keV}$ and above $20 \mathrm{keV}$ show a systematic deviation, and the $\chi^{2}$ is very poor.

The ionization parameter in both spectra is $\sim 40 \mathrm{erg} \mathrm{cm} \mathrm{s}^{-1}$. For such a value, according to Matt et al. (1993), the disc is expected to contribute a neutral iron line of about $100 \mathrm{eV}$. Indeed, 

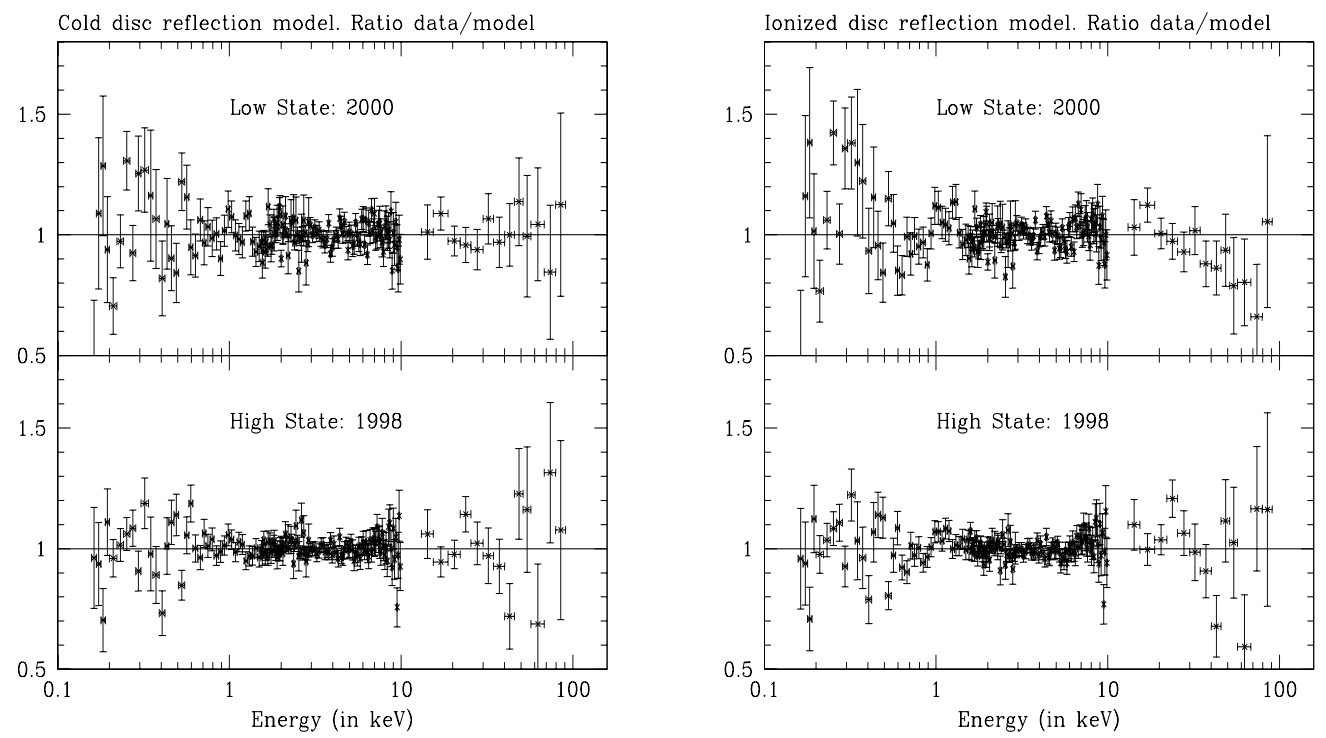

Fig. 3. Data/model ratio when the spectra in the low (upper panels) and high (lower panels) flux states of Mkn 509 are reproduced with a cold (left) and ionized (right) disc reflection model. See Tables 3 and 4 and for best fit parameters.

MKN 509. BeppoSAX

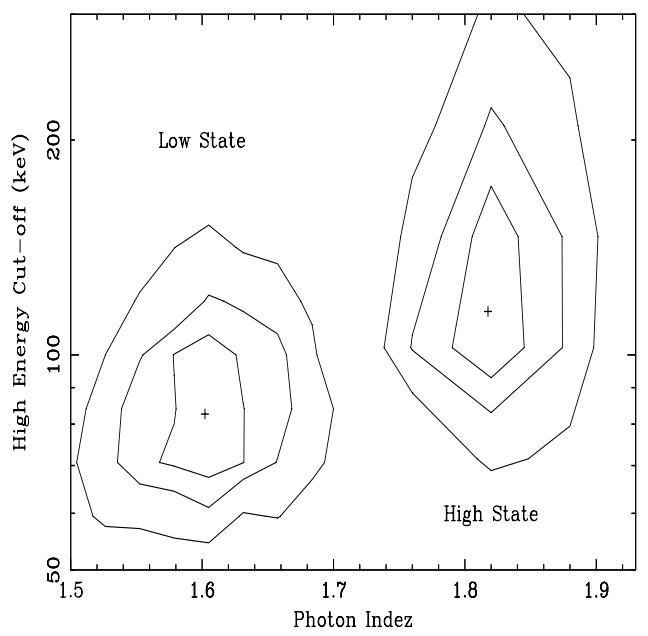

Mkn 509. BeppoSAX

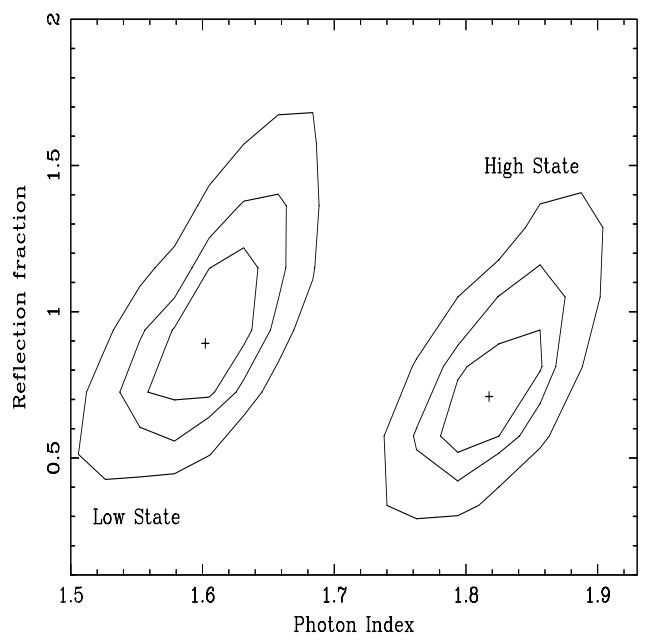

Fig. 4. $1 \sigma, 2 \sigma$ and $3 \sigma$ contour plots high energy cut-off vs. $\Gamma$ (left panel) and $R$ vs. $\Gamma$ (right panel) in the two flux states when the spectra are fitted with a cold disc reflection model.

we find that the intensity of the narrow line is somewhat smaller than that measured in the cold disc model. Again, the intensity of the narrow line does not change from one observation to the other, as expected if the line is produced in distant matter.

To test the possibility that the distant matter responsible for the narrow line is optically thick, we added a constant cold reflection component. In the high flux spectrum the reflection at low energies does still account reasonably well for the soft excess and the fit remains acceptable $\left(\chi^{2} /\right.$ d.o.f. $\left.=158.5 / 142\right)$, although the cold component becomes dominant over the ionized one $\left(R_{\text {cold }}=1.1_{-0.25}^{+0.65}, R_{\text {ion }}=0.4 \pm 0.2\right)$, while in the low flux spectrum no improvement is attained at low energies and the fit is worse $\left(\chi^{2} /\right.$ d.o.f. $\left.=187 / 143\right)$.

We note that also with this model the fit experiences a change $\Delta \Gamma \sim 0.2$ (see Fig. 5 and Table 4 ).

On balance, it seems to us that the cold reflection model, with the addition of an independent soft-excess component, is better suited to describe the two observations than the model just described.

\section{Discussion}

\subsection{The intrinsic continuum}

The broad-band BeppoSAX spectra of Mkn 509 are dominated by a variable power law with an exponential cut-off. The $e$-folding energy is found within the range of values observed in other Sy 1s with BeppoSAX (Perola et al. 2002). This model is an approximation of a Comptonized spectrum. In fact one of the emission mechanisms that is believed to work in the central region of AGN is a two-phase model involving a hot corona emitting medium-hard X-rays by Comptonization and an optically thick layer that provides the soft photons to be Comptonized (Haardt \& Maraschi 1993). However, the heating mechanism of the electrons, the geometry of the disc-corona 

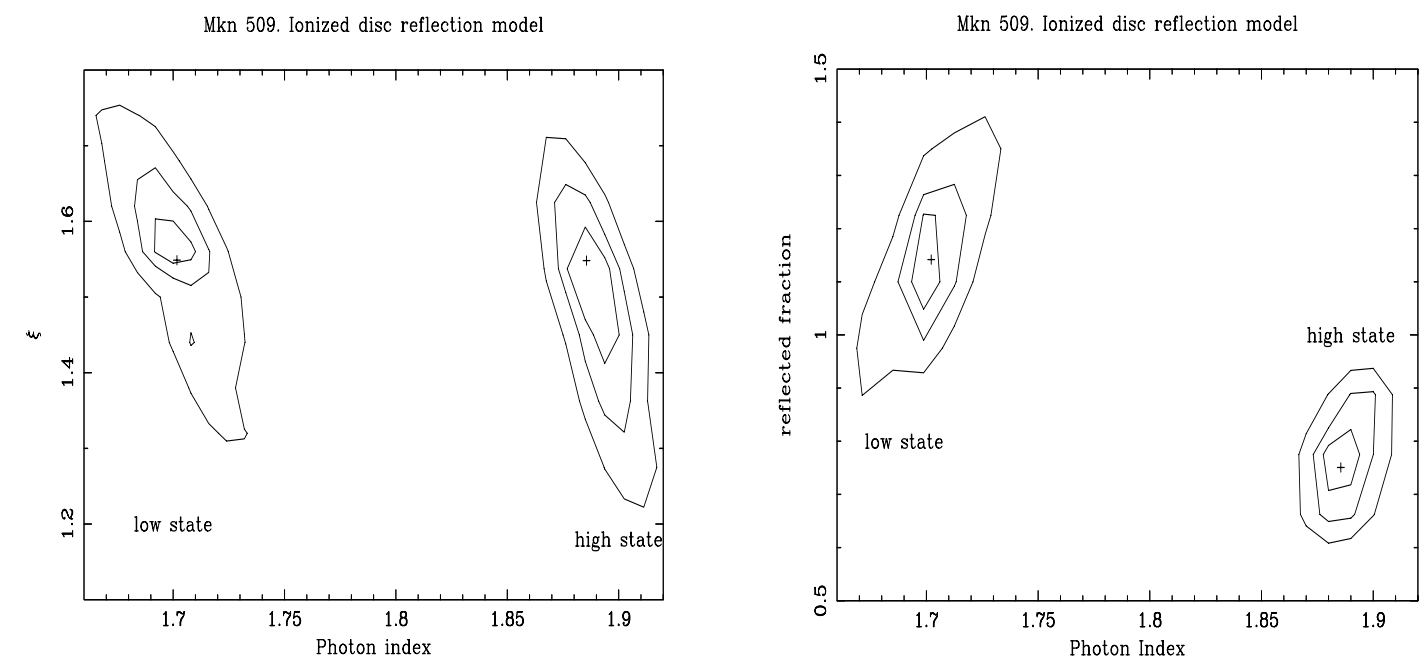

Fig. 5. Ionized disc reflection model (see Table 4). Confidence plots (68, 90 and 99 per cent) ionization parameter log $\xi$ vs. Photon index $\Gamma$ (left panel) and Reflected fraction vs. $\Gamma$ (right panel) in the different flux states of Mkn 509.

configuration and the origin of the variability are still very uncertain.

There is strong evidence that in Seyfert 1 galaxies the $\mathrm{X}$-ray spectrum softens as the $2-10 \mathrm{keV}$ flux increases (e.g. 1H 0419-577, Page et al. 2002; NGC 3783, De Rosa et al. 2002a, MCG-6-30-15, Vaughan \& Edelson 2001; NGC 5548, Petrucci et al. 2000, Nicastro et al. 2000; NGC 7469, Nandra et al. 2000; IC 4329A, Done et al. 2000, NGC 4151 Perola et al. 1986). Our analysis confirms this behaviour also in the case of Mkn 509, where a flux variation by a factor of two in the $2-10 \mathrm{keV}$ range is observed along with a change of the power law slope $\Delta \Gamma \sim 0.2$ (see Tables 3, 4 and Figs. 4, 5).

Analysing the spectral variability with a Comptonization model (Haardt \& Maraschi 1993) we found that an additional black body component was required (with $P_{\mathrm{F}}$ greater that 99.9 per cent), to model the soft X-ray spectra in both observations. The fit is still good in $1998\left(\chi^{2} /\right.$ d.o.f. $\left.=130 / 141\right)$, while this is not true in $2000\left(\chi^{2} /\right.$ d.o.f. $\left.=174 / 141\right)$, where large deviations are present below $1 \mathrm{keV}$ and above $40 \mathrm{keV}$. We found that for the high state the temperature of the corona and the Comptonization parameter $y$ (the Comptonized to soft luminosity ratio), are lower than in the low state: $k T_{\mathrm{e}}=65 \pm 10 \mathrm{keV}$, and $\tau=1.00_{-0.06}^{+0.08}$ in 1998 and $k T_{\mathrm{e}}=92_{-10}^{+20} \mathrm{keV}$ and $\tau=0.88_{-0.04}^{+0.06}$ in 2000. This behaviour, together with the fact that the continuum variation is higher at low energies (see lightcurves in Fig. 1), suggests an increase, in the high state, of the seed photons able to Comptonize the corona. This increase could be due either to some geometrical effect (e.g. a change of the inner radius of the disc) or to a change of the albedo of the disc (e.g. a variation of the ionization state). In both the hypotheses some variations in the strength of the reflection features are expected. However our analysis is consistent with a constant value of the reflection components (see discussion in Sect. 5.3). Then our analysis supports the idea that the observed variability is driven by a change in the soft photon flux which is not due to a change in either the geometry or the albedo of the disc.

\subsection{The soft $X$-ray excess}

As indicated by XMM-Newton and BeppoSAX observations, the soft excess is a common component in Seyfert 1 spectra (see e.g. Pounds \& Reeves 2001). Whether this component represents thermal emission directly from the accretion disc, or the result of the disc reprocessing of the hard X-rays impinging upon it, is still unclear. In a study of a small sample of Sy 1s observed by XMM-Newton, Pounds \& Reeves (2001) conclude that the "gradual soft excess" (GSX) detected in Mkn 509 could be interpreted as Comptonized thermal disc emission, this view being supported by the absence of discrete spectral features in RGS data. In addition they suggest that this broad soft emission component might be a common feature, which can sometimes be obscured by a warm absorber.

The analysis of the two BeppoSAX spectra of Mkn 509 shows that the absolute strength of the soft excess is definitely higher in the high flux state. However when it is reproduced with two black body components, it accounts for $\sim 30$ per cent of the $0.25-2 \mathrm{keV}$ observed luminosity in $2000\left(L_{0.25-2 \mathrm{keV}}^{2000}=\right.$ $\left.9 \times 10^{43} \mathrm{erg} \mathrm{s}^{-1}\right)$ and less than 25 per cent in $1998\left(L_{0.25-2 \mathrm{keV}}^{199-2 \mathrm{keV}}=\right.$ $\left.2.4 \times 10^{44} \mathrm{erg} \mathrm{s}^{-1}\right)$. When the spectra are fitted with an ionized disc reflection model, the soft excess in the high flux level state (1998) can be completely accounted for by the reflection of the disc, with no additional components required. In the low flux level state (2000), instead, the stronger relative contribution of the soft excess is only partially accounted for by a larger value of the reflection fraction (from a disc surprisingly characterized by the same ionization parameter as in 1998), and the global fit can hardly be considered acceptable. Despite the attractive behaviour of this model in one of the two observations, the alternative model with a cold reflection and the soft additional component, at least from a statistical point of view, behaves well in both observations, and it offers a natural explanation for the presence of the narrow and cold iron line.

We furthermore note that the black body components do not represent a unique empirical model that provides a good fit of the soft excess. Perola et al. (2000) describe this component 

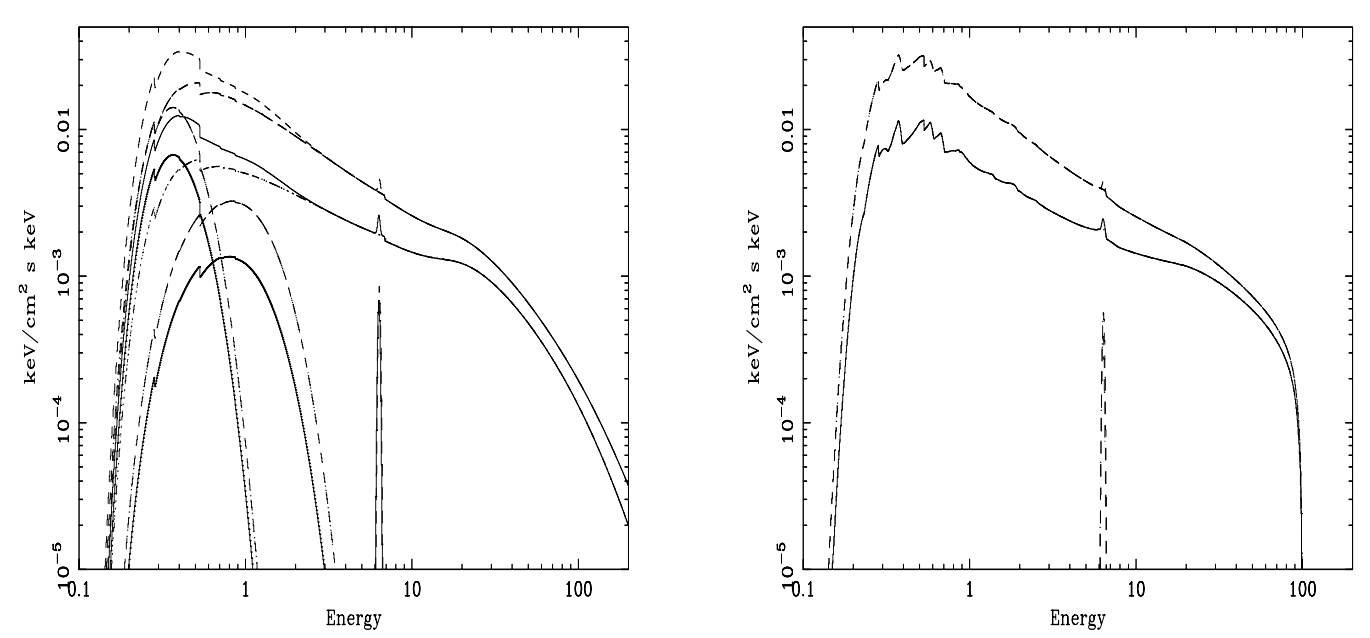

Fig. 6. The two different spectral models we checked, in the high (dash curves) and low (solid curves) flux state. In the left panel the cold disc reflection model is shown together with the black body components to reproduce the soft X-ray spectra. In the ionization disc reflection model (right panel) no additional component are required to model the spectra.

with a soft power law, and find that its extrapolation to lower energies matches the observed strength of the UV emission, admittedly observed in a different epoch. This lends some support to the idea that it could arise from Comptonization in the innermost part of the accretion disc (see also the case of NGC 5548 in Pounds et al. 2003).

\subsection{The reflection and fluorescent components}

Both the Compton reflection hump and the neutral iron emission line are detected in the BeppoSAX observations of Mkn 509. A single Gaussian component reproduces well the line profile. Perola et al. (2000) got similar results on the reflection features using a power law rather than two black body components to model the soft excess. The energy of the iron line they found is slightly higher than the value we report here, because in our broad-band fit we kept the intrinsic width of the line fixed to $0.1 \mathrm{keV}$.

The energy of the iron line indicates a low stage of ionization (FeI-XVII). Its intensity does not vary significantly between 1998 and 2000 and, moreover, it is consistent with that measured in a simultaneous Chandra HEG and RXTE PCA observation (Yaqoob \& Padmanabhan 2003) when the flux was similar to that observed by BeppoSAX in $1998, F_{2-10 \mathrm{keV}}^{\mathrm{Chanda}}=$ $5.5 \times 10^{-11} \mathrm{erg} \mathrm{cm}^{-2} \mathrm{~s}^{-1}$, and in a XMM-Newton observation in 2000 (Pounds et al. 2001). The amplitude of the reflection component is also consistent with a constant value in the two BeppoSAX observations, despite the variations in the primary flux. Thus it is most likely that both fluorescence and reflection take place at a distance of at least about one parsec from the central source.

A narrow component of the iron line is a common feature in Seyfert 1s (Padmanabhan \& Yaqoob 2003 and references therein). A recent analysis of a sample of nine Seyfert $1 \mathrm{~s}$ (Mkn 509 included) observed by XMM-Newton (Reeves 2003) found that the majority of the sources show an unresolved line at $6.4 \mathrm{keV}$. In general terms, given the typical EW found, this line is not necessarily produced by Compton thick material, for instance it could come from the Broad Line Region (which has typical column density of $10^{23} \mathrm{~cm}^{-2}$, Netzer 1990).

The case of Mkn 509, as assessed in this paper, adds to those of NGC 4051 (Guainazzi et al. 1998) and NGC 5506 (Matt et al. 2001), where a reflection component associated with a narrow cold iron line has indeed been detected.

Concerning the very marginal evidence we found for a ionized line in addition, it is worth mentioning the case of other objects where such, and generally weak, lines have been observed, like NGC 5506 (Matt et al. 2001), NGC 7213 (Bianchi et al. 2003), Mkn 205 (Reeves et al. 2001), NGC 3783 (De Rosa et al. 2002a), NGC 7469 (De Rosa et al. 2002b). As already mentioned, in the first XMM-Newton observation of Mkn 509 in 2000 Pounds et al. (2001) claimed the discovery of a weak ionized line in addition to the narrow cold line. After a reanalysis of the same observation, along with the analysis of a second one made in 2001, Page et al. (2003) reach the conclusion that illumination of a distant, cold and optically thick material is a simpler and self consistent explanation of the iron spectral features, rather than models including reflection from an ionized relativistic disc.

\section{Conclusions}

Taking advantage of two 0.1-100 keV BeppoSAX observations of Mkn 509, performed about two years apart, which found the source a factor of two different in the $2-10 \mathrm{keV}$ flux level, we could profitably compare two alternative models. The first model is a classical one with a cold Compton reflection, already investigated on the observation performed in 1998 by Perola et al. (2000), the other with the reflection from an ionized disc.

Common results of the two fitting models are:

a) The slope of the power law increases, $\Delta \Gamma \sim 0.2$, when the $2-10 \mathrm{keV}$ flux is a factor of two higher. This result agrees with the behaviour found in several other objects of this class and lends further support to the Comptonization models for the 
origin of the power law, in the form applied e.g. to NGC 5548 by Petrucci et al. (2000).

b) There is a cold iron fluorescent line, whose intensity and width are consistent with results obtained on the same object with XMM-Newton (Pounds et al. 2001; Page et al. 2003) and with a simultaneous Chandra HEG and RXTE PCA observation (Yaqoob \& Padmanabhan 2003). The stability in the intensity of this line, despite the rather large variations of the continuum level, speaks definitely in favour of a distant (of the order of one parsec) placement of the fluorescent material.

With respect to a simple power law, at low energies and in both observations there is a fairly strong and rather broad soft excess. The ionized disc reflection model can in principle account self-consistently for the existence of such an excess. And indeed, in the high state observation (1998) this model gives a fairly good fit, without requiring any extra component, except for the narrow line mentioned above. However, in the low state observation (2000) the outcome is a very poor fit, with substantial positive residuals below $1 \mathrm{keV}$. From a purely empirical point of view, this difference between the two states follows from the relative contribution of the soft excess being stronger when the source was in the low state, when the power law was at the same time harder.

With the cold reflection model, the soft excess must be modelled with an additional component, for which an empirical combination of two black bodies gives fair results in both states (an equally fair result was obtained on the first observation by Perola et al. 2000, adopting a soft power law). The luminosity of this excess in the $0.25-2 \mathrm{keV}$ range turns out to be about three times higher in the high (1998) state than in the low (2000) state. In our minds its origin and behaviour remains a substantially obscure issue.

The EW of the narrow line is modest and, in principle, could be explained as fluorescence from Compton thin material, for instance in the Broad Line Region. However a very important result with the cold reflection model is that the normalization of the Compton hump is perfectly consistent (within the large errors) with a constant value between the two epochs. This further argues in favour of a common origin of both the line and the reflection component in a distant Compton-thick material. The simultaneous measurement of both the line and the hump, which could be obtained with BeppoSAX and now with RXTE (alone or in a simultaneous combination with XMM-Newton and Chandra), adds Mkn 509 to the still short list of cases (NGC 5506, Matt et al. 2001; NGC 4051, Guainazzi et al. 1998) in which the above conclusion can be credibly reached.

\section{References}

Bianchi, S., Matt, G., Balestra, I., \& Perola, G. C. 1997, A\&A, 407, L21

Boella, G., Butler, R. C., Perola, G. C., et al. 1997, A\&A, 122, 299

De Rosa, A., Piro, L., \& Fiore, F. 2002a, A\&A, 387, 838

De Rosa, A., Fabian, A. C., \& Piro, L. 2002b, MNRAS, 334, L21

Done, C., Madejski, G. M., \& Zycki, P. T. 2000, ApJ, 536, 213

Fabian, A. C., Rees, M. J., Stella, L., \& White, N. E. 1989, MNRAS, 238, 729

Fabian, A. C., Iwasawa, K., Reynolds, C. S., \& Young, A. J. 2000, PASP, 112,1145
Fabian, A. C., Vaughan, S., Nandra, K., et al. 2002, MNRAS, 335, L1 Fiore, F., Guainazzi, G., \& Grandi, P. 1999, Cookbook for BeppoSAX NFI Spectral analysis. SDC report. (Available from http://asdc.asi.it/bepposax/software/index.html)

George, I., \& Fabian, A. C. 1991, MNRAS, 249, 352

George, I., Turner, T., Netzer, H., et al. 1998, ApJS, 114, 73

Ghisellini, F., Haardt, F., \& Matt, G. 1994, MNRAS, 267, 743

Guainazzi, M., Nicastro, F., Fiore, F., et al. 1998, MNRAS, 301, L1

Guainazzi, M., Matt, G., Molendi, S., et al. 1999, A\&A, 341, L27

Haardt, F., \& Maraschi, L. 1993, ApJ, 413, 507

Kaspi, S., Brandt, W. N., Netzer, H., et al. 2001, ApJ, 554, 216

Magdziarz, P., \& Zdziarski, A. A. 1995, MNRAS, 273, 837

Matt, G., Perola, G. C., \& Piro, L. 1991, A\&A, 247, 25

Matt, G., Fabian, A. C., \& Ross, R. R. 1993, MNRAS, 262, 179

Matt, G., Fabian, A. C., \& Ross, R. R. 1996, MNRAS, 278, 1111

Matt, G., Guainazzi, M., Perola, G. C., et al. 2001, A\&A, 377, L31

Matt, G., Guainazzi, M., \& Maiolino, R. 2003, MNRAS, 342, 422

Morini, M., Lipani, N., \& Molteni, D. 1987, ApJ, 17, 145

Murphy, E., Lockman, F., Laor, A., \& Elvis, M. 1996, ApJS, 105, 369

Nandra, K., George, I. M., Mushotzky, R. F., Turner, T. J., \& Yaqoob, T. 1997, ApJ, 477, 602

Nandra, K., Le, T., George, I., et al. 2000, ApJ, 544, 734

Netzer 1990, published in Active Galactic Nuclei, ed. R. D. Blandford, H. Netzer, \& L. Woltjer

Nicastro, F., Piro, L., De Rosa, A., et al. 2000, ApJ, 536, 718

Padmanabhan, \& Yaqoob 2003, in Conference Proceedings, High Energy Processes and Phenomena in Astrophysics, IAU Symp., 214, Suzhou, China, 2002

Page, K., Pounds, K., Reeves, J., \& O'Brien, P. 2002, MNRAS, 330, L1

Page, M. J., Davis, S. W., \& Salvi, N. J. 2003, MNRAS, accepted [astro-ph/0305043]

Perola, G. C., Piro, L., Altamore, A., et al. 1986, ApJ, 307, 486

Perola, G. C., Matt, G., Fiore, F., et al. 2000, A\&A, 358, 117

Perola, G. C., Matt, G., Cappi, M., et al. 2002, A\&A, 389, 802

Petrucci, P. O., Haardt, F., Maraschi, L., et al. 2000, ApJ, 540, 131

Piro, L., Nicastro, F., Feroci, M., et al. 1998, Nuclear Physics B (Proc. Suppl.), 69, 481

Pounds, K., Nandra, P., Fink, H., \& Makino, F. 1994, MNRAS, 267, 193

Pounds, K., Reeves, J., O’Brien, P., et al. 2001, ApJ, 559, 181

Pounds, K., \& Reeves, J. 2001, Proc. of the Symposium on New Visions of the X-ray Universe in the XMM-Newton and Chandra Era, ESTEC, The Netherlands [astro-ph/0201436]

Pounds, K., Reeves, J., Page, K., et al. 2003, MNRAS, 341, 953

Reeves, J. N., Turner, M., Pounds, K., et al. 2001, ApJ, 365, L134

Reeves, J. N. 2003, ASP Conf. Ser. [astro-ph/0211381]

Reynolds, C. 1997, MNRAS, 286, 513

Ross, R. R., \& Fabian, A. C. 1993, MNRAS, 261, 74

Singh, K. P., Garmire, G. P., \& Nousek, J. 1985, ApJ, 297, 633

Singh, K. P., Westergaard, N. J., Schnopper, H. W., Awaki, H., \& Tawara, Y. 1990, ApJ, 363, 131

Tanaka, Y, Nandra, K., Fabian, A. C., et al. 1995, Nature, 375, 659

Turner, T. J., Weaver, K. A., Mushotzky, R. F., Holt, S. S., \& Madejeski, G. M. 1991, ApJ, 381, 85

Vaughan, S., \& Edelson, R. 2001, ApJ, 548, 694

Yaqoob, T., George, I. M., Nandra, K., et al. 2001, ApJ, 546, 759

Yaqoob, T., Padmanabhan, U, Dotani, T., \& Nandra, K. 2002, ApJ, 569,487

Yaqoob, T., \& Padmanabhan, U. 2003, ASP Conf. Ser. [atro-ph/0211349]

Wilms, J., Reynolds, C. S., Begelman, M. C., et al. 2001, MNRAS, 328, L27 